Handbook of Zoology

Annelida

Volume 2:

Pleistoannelida, Sedentaria II 


\section{Handbook of Zoology}

Founded by Willy Kükenthal

continued by M. Beier, M. Fischer, J.-G. Helmcke, D. Starck, H. Wermuth Editor-in-chief Andreas Schmidt-Rhaesa

\section{Annelida}

Edited by Günter Purschke, Markus Böggemann and Wilfried Westheide 


\section{Annelida}

Volume 2:

Pleistoannelida, Sedentaria II

Edited by Günter Purschke, Markus Böggemann and Wilfried Westheide 


\section{Scientific Editors}

Prof. Dr. rer. nat. Günter Purschke

Universität Osnabrück

FB 5 - Biologie/Chemie

Barbarastr. 11

49076 Osnabrück

purschke@biologie.uni-osnabrueck.de

Prof. Dr. Markus Böggemann

Universität Vechta - Fakultät II

Natur- und Sozialwissenschaften/Biologie

Driverstr. 22

49377 Vechta

Markus.Boeggemann@uni-vechta.de

Herrn Prof. Dr. em. Wilfried Westheide

Gerhart-Hauptmann-Str. 3

49134 Wallenhorst

westheide@biologie.uni-osnabrueck.de

ISBN 978-3-11-029147-6

e-ISBN (PDF) 978-3-11-029168-1

e-ISBN (EPUB) 978-3-11-038884-8

ISSN 2193-4231

\section{Library of Congress Control Number: $\mathbf{9 7 8 3 1 1 0 2 9 1 4 7 6}$}

Bibliografic information published by the Deutsche Nationalbibliothek

The Deutsche Nationalbibliothek lists this publication in the Deutsche Nationalbibliografie; detailed bibliographic data are available in the Internet at http://dnb.dnb.de.

(C) 2019 Walter de Gruyter GmbH \& Co. KG, Berlin/Boston

Typesetting: XXX

Printing and Binding: $\mathrm{CPI}$ books $\mathrm{GmbH}$, Leck

www.degruyter.com 